\title{
Evaluation of a Fluorescent and Radiolabeled Hybrid Somatostatin Analog In Vitro and in Mice Bearing H69 Neuroendocrine Xenografts
}

\author{
Costanza Santini ${ }^{1}$, Joeri Kuil ${ }^{2}$, Anton Bunschoten ${ }^{2}$, Stefan Pool $^{1}$, Erik de Blois ${ }^{1}$, Yanto Ridwan ${ }^{3}$, Jeroen Essers ${ }^{3,4}$, \\ Monique R. Bernsen ${ }^{1}$, Fijs W.B. van Leeuwen ${ }^{2}$, and Marion de Jong ${ }^{1}$ \\ ${ }^{1}$ Department of Radiology and Nuclear Medicine, Erasmus MC, Rotterdam, The Netherlands; ${ }^{2}$ Interventional Molecular Imaging \\ Laboratory, Department of Radiology, Leiden University Medical Center, LUMC, Leiden, The Netherlands; ${ }^{3}$ Department of Genetics, \\ Erasmus MC, Rotterdam, The Netherlands; and ${ }^{4}$ Departments of Radiation Oncology and Vascular Surgery, Erasmus MC, Rotterdam, \\ The Netherlands
}

\begin{abstract}
In the treatment of neuroendocrine tumors (NETs), complete surgical removal of malignancy is generally desirable, because it offers curative results. Preoperative guidance with radiolabeled somatostatin analogs, commonly used for NET diagnosis and preoperative planning, is limited by its low resolution, with the risk that tumor margins and small metastases will be incompletely resected with subsequent recurrence. A single hybrid probe combining radiotracer and optical dye would enable high-resolution optical guidance, also during surgery. In the current study, the hybrid labeled somatostatin analog Cy5-DTPA-Tyr ${ }^{3}$-octreotate (DTPA is diethylene triamine pentaacetic acid) was synthesized and evaluated for its ability to specifically trace NET cells in vitro and in an animal model. The performance of the hybrid tracer was compared with that of octreotate with only radiolabel or only optical label. Methods: The binding affinity and internalization capacity of Cy5-DTPA-Tyr ${ }^{3}$ octreotate were assessed in vitro. Biodistribution profiles and both nuclear and optical in vivo imaging of Cy5- ${ }^{111} \mathrm{In}$-DTPA-Tyr ${ }^{3}$-octreotate were performed in NET-bearing mice and compared with the performance of ${ }^{111}$ In-DTPA-Tyr ${ }^{3}$-octreotate. Results: In vitro studies showed a low receptor affinity and internalization rate for Cy5DTPA-Tyr ${ }^{3}$-octreotate. The dissociation constant value was $387.7 \pm$ 97.9 nM for Cy5-DTPA-Tyr ${ }^{3}$-octreotate, whereas it was $120.5 \pm$ $18.1 \mathrm{nM}$ for DTPA-Tyr ${ }^{3}$-octreotate. Similarly, receptor-mediated internalization reduced from $33.76 \% \pm 1.22 \%$ applied dose for DTPA-Tyr ${ }^{3}$-octreotate to $1.32 \% \pm 0.02 \%$ applied dose for Cy5DTPA-Tyr ${ }^{3}$-octreotate. In contrast, in vivo and ex vivo studies revealed similar tumor uptake values of Cy5-111/n-DTPA-Tyr ${ }^{3}$-octreotate and ${ }^{111}$ In -DTPA-Tyr ${ }^{3}$-octreotate $(6.93 \pm 2.08$ and $5.16 \pm 1.27$, respectively). All organs except the kidneys showed low background radioactivity, with especially low activities in the liver, and high tumor-to-tissue ratios were achieved—both favorable for the tracer's toxicity profile. Hybrid imaging in mice confirmed that the nuclear and fluorescence signals colocalized. Conclusion: The correlation between findings with the optical and the nuclear probes underlines the potential of combining SPECT imaging with fluorescence guidance and shows the promise of this novel hybrid peptide for preoperative and intraoperative imaging of NET.
\end{abstract}

Received Aug. 10, 2015; revision accepted Mar. 17, 2016.

For correspondence or reprints contact: Costanza Santini, Department of Radiology and Nuclear Medicine, Rm. Na620, Erasmus MC, P.O. Box 2040, 3000 CA Rotterdam, The Netherlands.

E-mail: c.santini@erasmusmc.nl

Published online Apr. 28, 2016.

COPYRIGHT (c) 2016 by the Society of Nuclear Medicine and Molecular Imaging, Inc.
Key Words: image-guided surgery; octreotate; neuroendocrine tumors; multimodal imaging

J Nucl Med 2016; 57:1289-1295

DOI: 10.2967/jnumed.115.164970

$\mathbf{N}$ euroendocrine tumors (NETs) are rare neoplasms originating from endocrine cells and are known to overexpress the somatostatin receptor (sst). Of the 5 sst subtypes described, subtype 2 ( $\left.\mathrm{sst}_{2}\right)$ is the most commonly overexpressed in NET (1). Radiolabeled somatostatin peptide analogs, such as octreotide and $\mathrm{Tyr}^{3}$ octreotate, have a high affinity for the $\mathrm{sst}_{2}$ receptor (2). They have been used successfully in diagnosing patients with NET using nuclear imaging technologies, such as SPECT or PET, in combination with CT. They have also been used successfully for peptide receptor radionuclide therapy in patients with metastasized disease $(2,3)$, although surgical resection of the tumor tissue, if possible, is the preferred treatment for NET $(4,5)$.

Efficient tumor detection can considerably improve preoperative planning and result in better patient survival. In current clinical practice, ${ }^{68} \mathrm{Ga}$ and ${ }^{111} \mathrm{In}$ are common radiolabels used in the preoperative evaluation of lesions using PET- or SPECT-based imaging, respectively (6).

The introduction of portable $\gamma$-probes enabled real-time radioguidance during surgery (7-9). Nevertheless, radioguidance can neither determine tumor margins nor identify small metastases accurately $(4,10)$. The poor visual discrimination between tumor and healthy tissues raises the chance of an incomplete resection, associated with disease progression or recurrence (10).

Fluorescence imaging is an alternative technology for intraoperative guidance (11) that provides higher resolution and realtime optical detection of the tumor. However, photon attenuation limits tissue penetration to less than $1 \mathrm{~cm}$ (12); therefore, deep lesions cannot be identified based on fluorescence signal alone $(10,13,14)$.

The simultaneous use of optical and nuclear imaging modalities with their complementary strengths can improve surgical guidance and consequently resection outcome, as has been shown for sentinel node detection and resection (15). Dual-modality (or rather hybrid) radiocolloids offer 2 read-outs during surgery: the nuclear 
signal can roughly indicate the target location pre- and intraoperatively, and the fluorescence signal gives real-time submillimeter resolution during surgery, allowing accurate identification and precise resection.

Can this concept also be applied to tumor resection? Tumorspecific tracers, such as the peptide analogs mentioned above, are essential $(10,12)$, and 2 strategies can be followed. One strategy involves coinjection of 2 single-labeled targeting agents, that is, 1 agent carrying a fluorescent dye and 1 agent carrying a nuclear label. Alternatively, fluorescent and nuclear labels can both be attached to 1 targeting moiety, creating an imaging agent with 2 functionalities. The main drawback of the first strategy is the potentially different biodistribution profile of the 2 tracers, which could mean a mismatch between nuclear and optical signals (10). In contrast, the second strategy will provide automatic colocalization of the 2 signals. Such dual functionalization can be achieved by introducing 2 separate imaging labels onto each peptide or by introducing a single, hybrid label containing both functionalities (16). With the double-labeling approach, many previous studies with peptide-based tracers have demonstrated a substantial reduction in peptide-receptor affinity, including tracers based on somatostatin analogs $(16,17)$. However, hybrid label-modified tracers maintain a considerable level of receptor affinity both in vitro and in vivo (16-18). To the best of our knowledge, however, none of the hybrid-labeled somatostatin analogs has been tested for targeted imaging in vivo.

In this study, we investigated the tumor targeting potential of a newly synthetized somatostatin analog, the hybrid tracer Cy5-DTPA-Tyr ${ }^{3}$ octreotate (Fig. 1A), in vitro and in vivo. The tracer carries, on a single platform, the chelator diethylene triamine pentaacetic acid (DTPA), which chelates radiometals constituting the nuclear beacon, and the fluorescent cyanine dye Cy5 (excitation wavelength $=650 \mathrm{~nm}$; emission wavelength $=670 \mathrm{~nm}$ ) as optical label. This tracer was compared with the more traditional somatostatin analog DTPA-Tyr ${ }^{3}$-octreotate (Fig. 1B) and the fluorescently labeled Cy5 $\mathrm{Tyr}^{3}$-octreotate (Fig. 1C).

\section{MATERIALS AND METHODS}

\section{Synthesis}

Cy5-DTPA Hybrid Label. Cy5 was synthesized following a previously described procedure (19). The hybrid imaging label was synthesized using solid-phase peptide synthesis. After Fmoc deprotection (20\% piperidine in dimethylformamide [DMF]), Fmoc-Lys(IvDde)$\mathrm{OH}$ (2 equivalents [eq.]) was coupled overnight using benzotriazol-1yl-oxytripyrrolidinophosphonium hexafluorophosphate (PyBOP) (2 eq.), hydroxybenzotriazole (HOBt) (2 eq.), and $N, N$-diisopropylethylamine (DiPEA) (4 eq.) in DMF. After subsequent deprotection of the $\mathrm{N}$ terminus, DTPA ( $\mathrm{tBu})_{4}$ (1.3 eq.) was coupled overnight using PyBOP (1.3 eq.), HOBt (1.3 eq.), and DiPEA (3 eq.) in DMF. Next, the IvDde protecting group was removed with $2 \%$ hydrazine hydrate in DMF. Then Cy5 (1.3 eq.) was coupled overnight using PyBOP (1.3 eq.), HOBt (1.3 eq.), and DiPEA (10 eq.) in DMF. The hybrid label was deprotected and cleaved from the resin by treatment with trifluoroacetic acid (TFA) $(92.5 \%)$, ethylenediamine $(2.5 \%)$, TiS $(2.5 \%)$, and water $(2.5 \%)$ for $2 \mathrm{~h}$. Subsequently, the compound was precipitated in cold methyl-tert-butyl-ether/hexane (1:1), dissolved in $20 \% \mathrm{CH}_{3} \mathrm{CN}$ in $\mathrm{H}_{2} \mathrm{O}$, and lyophilized. The crude intermediate was used for the next reaction, in which a reactive linker was introduced. $N$-succinimidyl-4maleimidobutyrate (2 eq.) was reacted with the free thiol of the cysteine in dimethylsulfoxide with DiPEA (4 eq.). Thirty milligrams of the crude product were purified by reversed-phase high-performance liquid chromatography (HPLC) using a gradient of $0.1 \%$ TFA in $\mathrm{H}_{2} \mathrm{O} / \mathrm{CH}_{3} \mathrm{CN}$

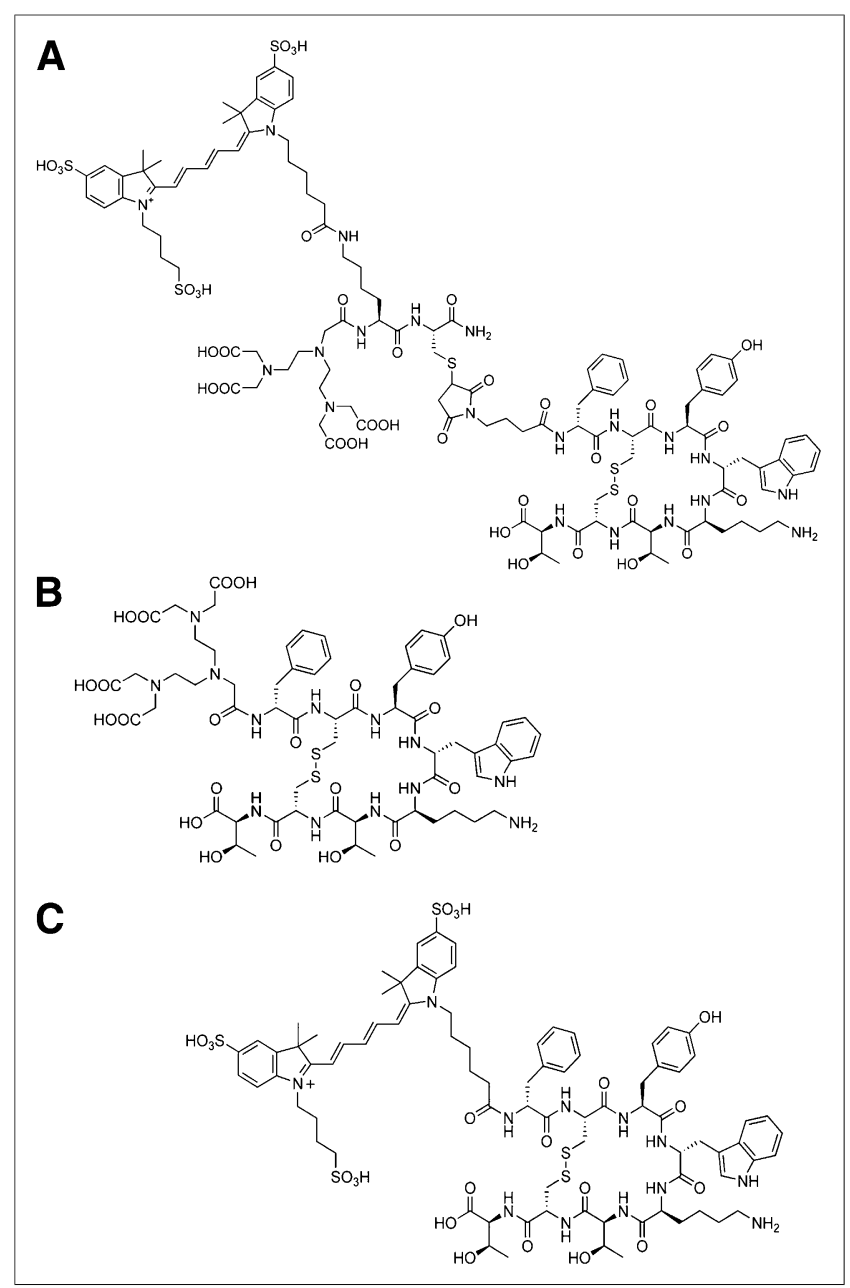

FIGURE 1. Structures of Cy5-DTPA-Tyr ${ }^{3}$-octreotate (A), DTPA-Tyr ${ }^{3}-$ octreotate (B), and Cy5-Tyr ${ }^{3}$-octreotate (C).

95:5\%-0.1\% TFA in $\mathrm{H}_{2} \mathrm{O} / \mathrm{CH}_{3} \mathrm{CN}$ 5:95 in 40 min. Fractions containing the right mass were pooled and lyophilized to yield $9.5 \mathrm{mg}$ of a blue fluffy solid. MS: $[\mathrm{M}+\mathrm{H}]^{+}$calculated $1,650.5$, found $1,651.5$.

Synthesis of DTPA-Tyr ${ }^{3}$-Octreotate, Cy5-Tyr ${ }^{3}$-Octreotate, and Cy5DTPA-Tyr ${ }^{3}$-Octreotate. Resin-bound D-Phe-Cys(Acm)-Tyr(tBu)-D$\operatorname{Trp}(\mathrm{Boc})-\mathrm{Lys}(\mathrm{Boc})-\operatorname{Thr}(\mathrm{tBu})-\mathrm{Cys}(\mathrm{Acm})-\operatorname{Thr}(\mathrm{tBu})(\mathbf{1})$ was synthesized as previously described $(20)$ and further specified in the supplemental materials (available at http://jnm.snmjournals.org). Peptidyl resin $1(25 \mu \mathrm{mol})$ was swollen in $\mathrm{CH}_{2} \mathrm{Cl}_{2}$. Depending on the peptide under synthesis, different concentrations of DTPA-tetra (t-Bu ester), Cy5, or Cy5-DTPA hybrid label were added to the mixture with different concentrations of peptide coupling agents in DMF. The mixture was stirred overnight at room temperature. The resin was then washed with $\mathrm{DMF}(3 \times)$ and $\mathrm{CH}_{2} \mathrm{Cl}_{2}(3 \times)$ and dried under vacuum. DTPA-Tyr ${ }^{3}$-octreotate, Cy5-Tyr ${ }^{3}$-octreotate, or Cy5DTPA-Tyr ${ }^{3}$-octreotate (as appropriate) was cleaved from the resin, and the side chains were deprotected with a solution of TFA/ $\mathrm{H}_{2} \mathrm{O} / \mathrm{TIS}$ 90:5:1.5 $(5 \mathrm{~mL})$ for $3 \mathrm{~h}$. The resin was removed from the solution by filtration. The peptides were precipitated with MTBE/hexane $1: 1 \mathrm{v} / \mathrm{v}$ at $-20^{\circ} \mathrm{C}$ and lyophilized from $\mathrm{CH}_{3} \mathrm{CN} / \mathrm{H}_{2} \mathrm{O} 1: 1 \mathrm{v} / \mathrm{v}$, yielding $21.7 \mathrm{mg}$ of crude peptide for DTPA-Tyr ${ }^{3}$-octreotate, $25.7 \mathrm{mg}$ for Cy5-Tyr ${ }^{3}$-octreotate, and $25.5 \mathrm{mg}$ for Cy5-DTPA-Tyr ${ }^{3}$-octreotate. The peptides were purified by preparative HPLC using a gradient of $0.1 \%$ TFA in $\mathrm{H}_{2} \mathrm{O}$ / $\mathrm{CH}_{3} \mathrm{CN}$ 9:1\%-0.1\% TFA in $\mathrm{H}_{2} \mathrm{O} / \mathrm{CH}_{3} \mathrm{CN}$ 1:9 in 60 min (DTPA-Tyr ${ }^{3}$ octreotate, Cy5-Tyr ${ }^{3}$-octreotate) or $120 \mathrm{~min}$ (Cy5-DTPA-Tyr ${ }^{3}$-octreotate). 
After pooling of the appropriate fractions and lyophilization, $4.4 \mathrm{mg}$ of pure DTPA-Tyr ${ }^{3}$-octreotate, $6.8 \mathrm{mg}$ of pure Cy5-Tyr ${ }^{3}$-octreotate, and $1 \mathrm{mg}$ of pure Cy5-DTPA-Tyr ${ }^{3}$-octreotate were obtained. Further details can be found in the supplemental materials.

Tyr $r^{3}$-Octreotate. Tyr $^{3}$-octreotate was purified from the crude yield of Cy5-DTPA-Tyr ${ }^{3}$-octreotate. The peptide was isolated with preparative HPLC using a gradient of $0.1 \%$ TFA in $\mathrm{H}_{2} \mathrm{O} / \mathrm{CH}_{3} \mathrm{CN} 9: 1 \%-0.1 \%$ TFA in $\mathrm{H}_{2} \mathrm{O} / \mathrm{CH}_{3} \mathrm{CN}$ 1:9 in $180 \mathrm{~min}$.

\section{Labeling with ${ }^{111}$ In and ${ }^{125}$ I}

For the binding affinity study, $\mathrm{Tyr}^{3}$-octreotate was labeled with ${ }^{125} \mathrm{I}$ following the chloramine-T method as previously described (21). For ${ }^{111}$ In radiolabeling, DTPA-Tyr ${ }^{3}$-octreotate or Cy5-DTPA-Tyr ${ }^{3}$-octreotate was mixed with ${ }^{111} \mathrm{InCl}_{3}$ (1 eq.) and dissolved in $0.1 \mathrm{M}$ of acetic acid. Labeling was performed as previously described (22) and further specified in the supplemental materials.

\section{In Vitro Studies}

Fluorescence-Based Flow Cytometry and Binding Assay. The dissociation constant $\left(\mathrm{K}_{\mathrm{D}}\right)$ was determined by a fluorescence-based flow cytometry assay described previously (23). C204 Luc 189 RR cells were trypsinized and aliquoted in portions of 300,000 cells. For saturation binding experiments, each aliquot received $50 \mu \mathrm{L}$ of $0.1 \%$ bovine serum albumin (BSA) in phosphate-buffered saline (PBS), containing concentrations of Cy5-Tyr ${ }^{3}$-octreotate, Cy5-DTPA-Tyr ${ }^{3}$-octreotate, or Cy5-In-DTPA-Tyr ${ }^{3}$-octreotate ranging between 0 and $10^{3} \mathrm{nM}$. For competition binding experiments, each aliquot received $50 \mu \mathrm{L}$ of $0.1 \%$ BSA in PBS containing $100 \mathrm{nM}$ of Cy5-DTPA-Tyr ${ }^{3}$-octreotate as well as $10^{-1}-3 \times 10^{4} \mathrm{nM}^{-} \mathrm{Tyr}^{3}$-octreotate, DTPA-Tyr ${ }^{3}$-octreotate, or In-DTPA-Tyr ${ }^{3}$-octreotate. Cells were incubated for $1 \mathrm{~h}$ at $4^{\circ} \mathrm{C}$, after which they were washed twice with $300 \mu \mathrm{L}$ of $0.1 \%$ BSA in PBS and resuspended in $300 \mu \mathrm{L}$ of $0.1 \%$ BSA in PBS. Fluorescence was measured using a BD FACSCanto II flow cytometer (BD Biosciences) with APC settings (633 nm laser, 660/20 nm filter). Live cells were gated on a forward scatter/side scatter plot, and 20,000 live cells were analyzed. All experiments were performed in duplicate.

Internalization Study. Rat pancreatic $\mathrm{sst}_{2}$-expressing CA20948 tumor cells were isolated as previously described (24) and seeded in 6-well plates $\left(0.5 \times 10^{6}\right.$ cells/well $)$. After $24 \mathrm{~h}$, cells were incubated with Cy5- ${ }^{111}$ In-DTPA-Tyr ${ }^{3}$-octreotate or ${ }^{111}$ In-DTPA-Tyr ${ }^{3}$-octreotate for $1 \mathrm{~h}$ at $37^{\circ} \mathrm{C}$ (per well: $0.035 \mathrm{nmol}, 100 \mathrm{MBq} / \mathrm{nmol}$ ). The specific binding was assessed for both tracers by coincubation with a 100 -fold molar excess of unlabeled peptide. The data were analyzed as previously described (25).

\section{In Vivo/Ex Vivo Studies}

Tumor Model. Human small cell lung cancer H69 cells (ECACC) were cultured under standard conditions in Gibco RPMI medium (Invitrogen) supplemented with $10 \% \mathrm{v} / \mathrm{v}$ fetal bovine serum and $1 \% \mathrm{v} / \mathrm{v}$ penicillin/streptomycin. Before tumor inoculation, $2.3 \times 10^{6}$ cell $/ \mathrm{mL}$ were suspended in 2:1 v/v Seligmann buffered salt solution medium and Matrigel (BD Biosciences). The suspension was inoculated subcutaneously in 25 young adult male BALB/c- $\nu$-mice (Janvier) (100 $\mu \mathrm{L} /$ mouse). Tumor growth was monitored 3 times/wk. When tumors reached approximately $300 \mathrm{~mm}^{3}$, animals were divided into 4 groups with similar distributions of tumor volumes: 2 groups of 8 animals for nuclear investigations and 2 of 4 or 5 mice each for optical and multimodal studies.

All animal experiments were performed in accordance with Dutch animal welfare regulations and approved by the local ethics committee.

SPECT/CT Imaging and Nuclear-Based Biodistribution. Eight mice were given an intravenous injection of Cy5-111 In-DTPA-Tyr ${ }^{3}$-octreotate (per animal: $200 \mathrm{pmol}, 30 \mathrm{MBq}, 200 \mu \mathrm{L}$ ); another 8 were given an injection of ${ }^{111} \mathrm{In}$-DTPA-Tyr ${ }^{3}$-octreotate (per animal: 200 pmol, $30 \mathrm{MBq}$,
$200 \mu \mathrm{L}$ ). Animals were anesthetized with a mixture of isoflurane (Pharmachemie BV; $4 \%$ for induction and $1.5 \%$ for maintenance) and oxygen and scanned for $30 \mathrm{~min}$ with SPECT/CT at 2 and $24 \mathrm{~h}$ after injection (NanoSPECT/CT; Bioscan). Further information is available in the supplemental materials.

After the last scan, animals were euthanized by cervical dislocation, and blood was immediately collected by heart puncture. Several organs and the tumor were harvested and weighed. Radioactivity was measured with a $\gamma$-counter (Wallac, 1480 Wizard 3"; PerkinElmer). Uptake was calculated as percentage injected dose per gram $(\% \mathrm{ID} / \mathrm{g})$.

Optical-Based and Multimodal Imaging. For optical and multimodal analyses, 5 mice were injected with Cy5-111 In-DTPA-Tyr ${ }^{3}$ octreotate (per animal: 200 pmol, $30 \mathrm{MBq}, 200 \mu \mathrm{L}$ ) and another 4 mice with Cy5-Tyr ${ }^{3}$-octreotate (per animal 200 pmol, $200 \mu \mathrm{L}$ ). All mice were imaged at $24 \mathrm{~h}$ after injection, using a fluorescence tomography device (FMT, 2500XL; PerkinElmer Inc.). The mice injected with Cy5-Tyr ${ }^{3}$-octreotate were euthanized immediately after the fluorescence tomography scan, the skin around the tumor was removed, and the tumor was scanned with an IVIS imaging system (Perkin Elmer). The mice injected with Cy5-111 In-DTPA-Tyr ${ }^{3}$ octreotate underwent SPECT/CT scanning at $24 \mathrm{~h}$ after injection before being euthanized. Further information is available in the supplemental materials.

\section{Data Analysis and Statistical Methods}

All data collected were processed with Prism version 5 (GraphPad Software). A 2-tailed Student $t$ test was used, and a $P$ value of less than 0.05 was considered statistically significant.

\section{RESULTS}

\section{Binding Affinity}

Data obtained from the fluorescence-based binding assays were used to generate concentration-binding curves of the various compounds (Fig. 2) and to calculate their $\mathrm{K}_{\mathrm{D}}$ and inhibitory concentration of $50 \%\left(\mathrm{IC}_{50}\right)$ values. More details are provided

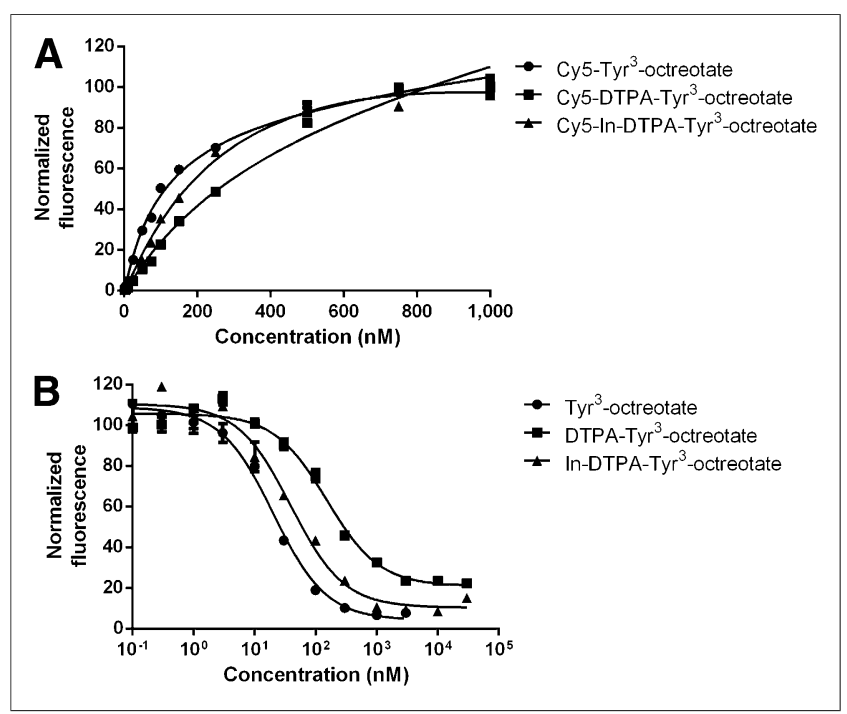

FIGURE 2. (A) Fluorescence-based flow cytometry saturation binding assay of Cy5-Tyr ${ }^{3}$-octreotate, Cy5-DTPA-Tyr ${ }^{3}$-octreotate, and Cy5-InDTPA-Tyr ${ }^{3}$-octreotate. (B) Fluorescence-based flow cytometry competition binding assay. Cy5-DTPA-Tyr ${ }^{3}$-octreotate was used as ligand to be displaced at increasing concentration of $\mathrm{Tyr}^{3}$-octreotate, DTPA-Tyr ${ }^{3}$ octreotate, and In-DTPA-Tyr ${ }^{3}$-octreotate. 
TABLE 1

Receptor Binding Parameters of Different Tracers

\begin{tabular}{lcc}
\hline \multicolumn{1}{c}{ Tracer } & $\mathrm{K}_{\mathrm{D}} \pm \mathrm{SD}(\mathrm{nM})$ & $\mathrm{IC}_{50}(\mathrm{nM})$ \\
\hline Tyr ${ }^{3}$-octreotate & $16.2 \pm 1.6$ & $20.35(16.8-24.7)$ \\
DTPA-Tyr ${ }^{3}$-octroetate & $120.5 \pm 18.1$ & $151.5(112.6-204.0)$ \\
In-DTPA-Tyr ${ }^{3}$-octreotate & $31.2 \pm 5.4$ & $39.3(28.0-55.1)$ \\
Cy5-Tyr ${ }^{3}$-octreotate & $106.1 \pm 11.7$ & $133.4(107.5-159.5)$ \\
Cy5-DTPA-Tyr ${ }^{3}$-octreotate & $387.7 \pm 97.9$ & $487.7(400.6-574.8)$ \\
Cy5-In-DTPA-Tyr ${ }^{3}$-octreotate & $415.0 \pm 90.0$ & $522.0(399.2-644.9)$ \\
& & \\
\hline Data in parentheses are 95\% confidence intervals (nM). & & \\
\hline
\end{tabular}

in Table 1 and the supplemental materials. On the basis of both $\mathrm{K}_{\mathrm{D}}$ and $\mathrm{IC}_{50}$ values, we observed a reduced receptor binding affinity for the peptide derivatives tested: unlabeled $\mathrm{Tyr}^{3}$ octreotate $>$ DTPA-Tyr ${ }^{3}$-octreotate $>$ Cy5- ${ }^{111}{ }^{1}$ In-DTPA-Tyr ${ }^{3}$ octreotate.

\section{In Vitro Tumor Uptake}

The internalization efficacy of Cy5-111 In-DTPA-Tyr ${ }^{3}$-octreotate was assessed in comparison to the nuclear-only tracer ${ }^{111} \mathrm{In}$ DTPA-Tyr ${ }^{3}$-octreotate (Fig. 3). First, the total uptake, measured as fraction of the applied dose (AD), that was retained in the cells was evaluated. For ${ }^{111} \mathrm{In}$-DTPA-Tyr ${ }^{3}$-octreotate and Cy5- ${ }^{111} \mathrm{In}$ DTPA-Tyr ${ }^{3}$-octreotate, the total uptake was $33.76 \% \pm 1.22 \%$ $\mathrm{AD}$ and $1.32 \% \pm 0.02 \% \mathrm{AD}$, respectively (Fig. 3).

Second, we distinguished between the internalized (cell fraction) and the membrane-bound fraction (membrane fraction). For ${ }^{111}$ In-DTPA-Tyr ${ }^{3}$-octreotate, the cell fraction and the membrane fractions were $30.76 \% \pm 1.02 \% \mathrm{AD}$ and $3.49 \% \pm 0.3 \% \mathrm{AD}$, respectively, whereas the values for Cy5- ${ }^{111}$ In-DTPA-Tyr ${ }^{3}$-octreotate were $1.21 \% \pm 0.03 \% \mathrm{AD}$ and $0.12 \% \pm 0.001 \% \mathrm{AD}$, respectively. Coincubation with an excess of DTPA-Tyr ${ }^{3}$-octreotate or Cy5DTPA-Tyr ${ }^{3}$-octreotate unlabeled peptide (block) significantly reduced total uptake to $0.15 \%$ AD for ${ }^{111} \mathrm{In}$-DTPA-Tyr ${ }^{3}$-octreotate and to $0.04 \% \mathrm{AD}$ for $\mathrm{Cy} 5-{ }^{111} \mathrm{In}$-DTPA-Tyr ${ }^{3}$-octreotate, indicating receptor specificity for both tracers (Fig. 3).

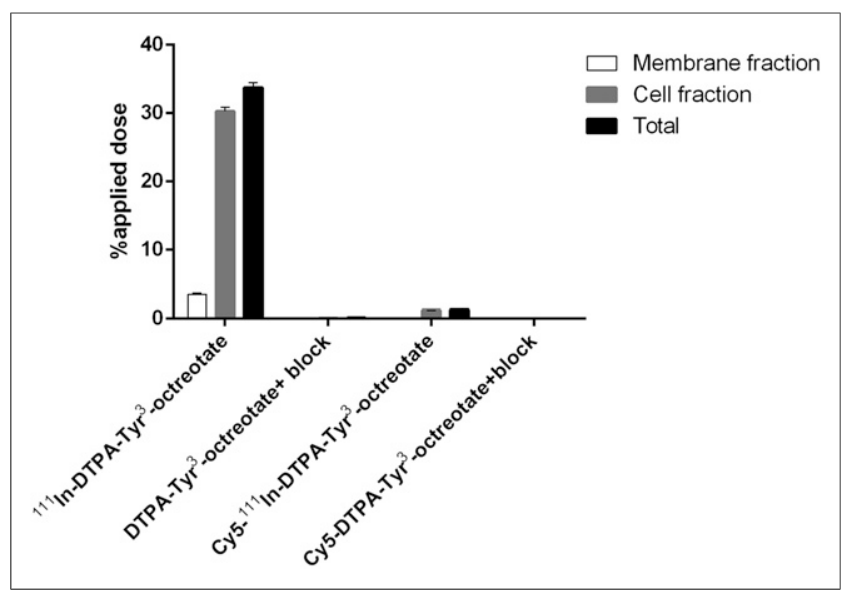

FIGURE 3. Uptake of ${ }^{111} \mathrm{In}$-DTPA-Tyr ${ }^{3}$-octreotate and Cy5-111/nDTPA-Tyr ${ }^{3}$-octreotate by CA20948 cells.
Calculation of the percentage of internalized tracer versus the total uptake gave similar values for both tracers (Table 2), revealing that the 2 tracers have similar kinetics once bound to the receptor.

\section{In Vivo/Ex Vivo: Tumor Uptake and Nuclear-Based Biodistribution}

SPECT/CT images at different time points indicate highly similar in vivo behavior of ${ }^{111}{ }^{1 n}$-DTPA-Tyr ${ }^{3}$-octreotate and Cy5-111 In-DTPA-Tyr ${ }^{3}$-octreotate, both showing substantial uptake in the tumor and in the kidneys but generally low radioactivity in background organs (Fig. 4). Interestingly, radioactivity was not detectable in the liver, indicating low hepatic retention for both tracers. In vivo quantification of kidney and tumor radioactivity is shown in Figure 4E: the data confirmed Cy5-DTPA-Tyr ${ }^{3}$-octreotate accumulation in both the kidneys and the tumor.

Ex vivo biodistribution data of ${ }^{111}$ In-DTPA-Tyr ${ }^{3}$-octreotate and Cy5-111 In-DTPA-Tyr ${ }^{3}$-octreotate agreed with the pattern observed with the SPECT/CT scans, showing low accumulation $(<2 \% \mathrm{ID} / \mathrm{g})$ in organs other than the kidneys (Table 3). Noticeably, liver levels were extremely low. For the kidneys, a high and consistent retention was visible: $14.25 \pm 2.79 \% \mathrm{ID} / \mathrm{g}$ for ${ }^{111}{ }^{1}$ In-DTPA-Tyr ${ }^{3}$-octreotate and $52.21 \pm 7.85 \% \mathrm{ID} / \mathrm{g}$ for Cy5- ${ }^{111} \mathrm{In}$-DTPA-Tyr ${ }^{3}$-octreotate. Despite the fact that receptor affinity of the hybrid tracer was significantly reduced as measured in vitro, tumor uptake in vivo was relatively high for both tracers (mean, $\sim 5 \% \mathrm{ID} / \mathrm{g}$ ) and not statistically different $(P>0.05)$. The calculated tumor-to-tissue ratios (TTRs) — with the exception of the kidneys—-were always greater than 3 and usually greater than 10 (Table 3), indicating that tumor specificity was high.

\section{Optical-Based Analyses}

Tumor uptake was quantified based on the FMT signal, and the results are depicted in Figure 5A. Optical signal originating from Cy5-111 In-DTPA-Tyr ${ }^{3}$-octreotate and Cy5-Tyr ${ }^{3}$-octreotate is comparable at $24 \mathrm{~h}$ after injection. The optical image shown in Figure $5 \mathrm{~B}$ was obtained with an IVIS imaging system. The image shows a representative mouse of those injected with fluorescently labeled octreotate and indicates the potential of visualizing tumor tissue and margins in vivo using the fluorescence signal.

\section{Multimodal Analyses with Cy5-111 In-DTPA-Tyr ${ }^{3}$-Octreotate}

The combined optical and nuclear imaging capability of Cy5- ${ }^{111}$ In-DTPA-Tyr ${ }^{3}$-octreotate is shown in Figure 6. The images indicate that tracer accumulation in the tumor can be detected with both nuclear and optical imaging modalities. 
TABLE 2

Radioactivity in Membrane Fraction and Cell Fraction ${ }^{111}$ In-DTPA-Tyr ${ }^{3}$-Octreotate and Cy5-111 In-DTPA-Tyr ${ }^{3}-$ Octreotate

\begin{tabular}{lcc}
\hline \multicolumn{1}{c}{ Tracer } & Mean membrane fraction \pm SD $(\%$ total uptake $)$ & Mean cell fraction \pm SD $(\%$ total uptake $)$ \\
\hline${ }^{111}$ In-DTPA-Tyr ${ }^{3}$-octreotate & $10.33 \pm 0.67$ & $89.67 \pm 0.70$ \\
Cy5-111 In-DTPA-Tyr ${ }^{3}$-octreotate & $8.86 \pm 0.97$ & $91.14 \pm 0.97$ \\
\hline
\end{tabular}

\section{DISCUSSION}

In NETs, in which radical tumor resection is often the only curative option, more refined surgical guidance, with the possibility of real-time tumor margin identification, would be highly beneficial for patient survival (7). Combined fluorescence and nuclear imaging represents an attractive approach to improve guidance because it allows for combined pre- and intraoperative imaging, with high resolution and real-time feedback provided by fluorescence imaging. The correlation we found between optical and nuclear imaging confirms that the combination of the 2 techniques is a promising approach for pre- and intraoperative imaging in NET.

One of the main drawbacks of dual-labeled tracers is the potential loss of receptor affinity and in vivo targeting capability, which has previously been reported for various dual-labeled tracers $(16,17,26,27)$. Cy5- ${ }^{111}$ In-DTPA-Tyr ${ }^{3}$-octreotate and ${ }^{111}$ In-DTPA-Tyr ${ }^{3}$-octreotate
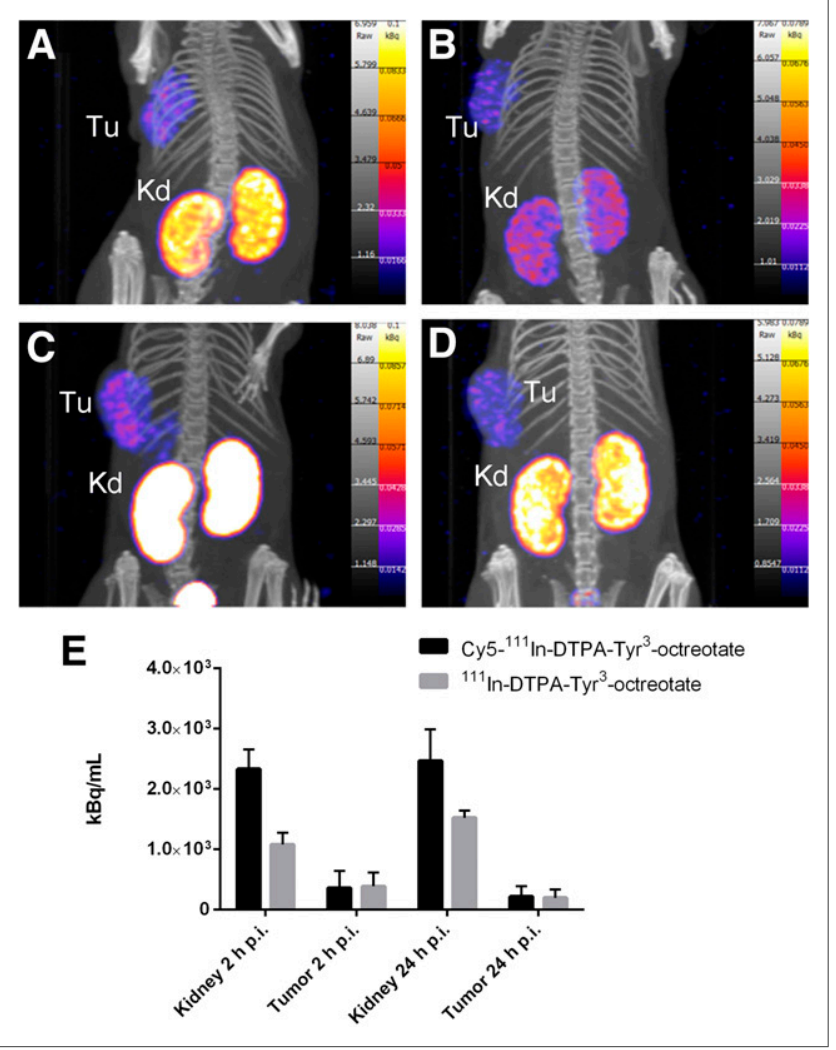

FIGURE 4. SPECT/CT images of H69 tumor-bearing mice. ${ }^{111} / \mathrm{n}$ DTPA-Tyr ${ }^{3}$-octreotate at 2 (A) and $24 \mathrm{~h}(\mathrm{~B})$ after injection and Cy5-111/n-DTPA-Tyr ${ }^{3}$-octreotate at 2 (C) and $24 \mathrm{~h}$ (D) after injection. (E) Quantification of tumor and kidney 2 and $24 \mathrm{~h}$ after injection. Mean for $n=8$ of calculated uptake \pm SD. Kd = kidney; p.i. = after injection; $\mathrm{Tu}=$ tumor. showed comparable profiles when applied in our in vivo model, especially with respect to tumor uptake and limited off-target uptake; the only major difference between ${ }^{111} \mathrm{In} \mathrm{DTPA}-\mathrm{Tyr}^{3}$ octreotate and Cy5-111 In-DTPA-Tyr ${ }^{3}$-octreotate was the renal uptake, which was higher with the dual-labeled tracer than with the nuclear-only tracer. It is known that ${ }^{111} \mathrm{In}$-DTPA-Tyr ${ }^{3}$-octreotate is cleared via and partially retained in the kidneys as a result of renal reabsorption in the proximal tubular cells, mostly due to the tracer's molecular composition (28). We hypothesized that the 3 additional negative charges present on Cy5 contributed to the increased renal retention of Cy5- ${ }^{111}$ In-DTPA-Tyr ${ }^{3}$-octreotate. A favorable finding concerning the hybrid Cy5-111 In-DTPA-Tyr ${ }^{3}$ octreotate is the low level of liver uptake, which was comparable to that of ${ }^{111} \mathrm{In}$-DTPA-Tyr ${ }^{3}$-octreotate. High hepatic retention has been reported for several other hybrid tracers $(16,18)$. Such in vivo behavior was explained as a result of the hydrophobic nature of cyanine dye and the large noncovalent interaction with serum albumin that drives increased retention in all tissues, including the high hepatic uptake $(18,29)$. In our study, we used a relatively hydrophilic cyanine dye, specifically chosen to reduce the albumin interaction. The 3 negative charges, which are likely responsible for the increased renal retention, therefore also appear to contribute to the limited degree of hepatic clearance. Nonetheless, Cy5- ${ }^{111} \mathrm{In}-$ DTPA-Tyr ${ }^{3}$-octreotate was retained in the bloodstream for longer than the corresponding nuclear-only tracer; such behavior could be explained by an interaction with albumin (26). Indeed, in a pilot protein binding study, the serum protein fraction contained more than 30 times as much Cy5-111 In-DTPA-Tyr ${ }^{3}$-octreotate than the watery fraction of blood (data not shown). The longer circulation time might explain the improved tumor uptake, this longer circulation time resulting from reduced renal filtration or by degradation protection. This hypothesis requires further investigation before definitive conclusions can be drawn.

Regarding stability in the bloodstream, peptide molecules are known to be vulnerable to degradation by peptidases (30). Nonetheless, radiolabeled DTPA- and DOTA-linked $\mathrm{Tyr}^{3}$-octreotide and $\mathrm{Tyr}^{3}$-octreotate have exhibited excellent stability in circulation; they are excreted via the urine mostly in the intact form (30-32). Therefore, we expect our hybrid Cy5- ${ }^{111}$ In-DTPA-Tyr ${ }^{3}$-octreotate peptide analog to exhibit good stability in circulation as well. Indeed, the Cy5-111 In-DTPA-Tyr ${ }^{3}$-octreotate biodistribution profile remained comparable with that of ${ }^{111} \mathrm{In}$-DTPA-Tyr ${ }^{3}$-octreotate over time. Moreover, the hybrid probe uptake in tumor could be detected using both nuclear and fluorescent imaging modalities, indicating that the radiolabel and fluorophore on the hybrid probe were not separated during transit to the tumor.

Our results on the hybrid Cy5-111 In-DTPA-Tyr ${ }^{3}$-octreotate are particularly promising for application to image-guided surgery. For radio- or fluorescence-guided surgery, a significant difference in uptake by tumor and normal tissue is needed for discrimination. A TTR value of 1.5 is generally considered sufficient for radioguidance (7), whereas a TTR value of 3 is thought to be needed for 
TABLE 3

Biodistribution (in \%ID/g) and TTR of ${ }^{111} \mathrm{In}-\mathrm{DTPA}-\mathrm{Tyr}^{3}-$ Octreotate and Cy5-111 In-DTPA-Tyr ${ }^{3}$-Octreotate at 24 Hours After Injection

\begin{tabular}{|c|c|c|c|c|}
\hline \multirow[b]{2}{*}{ Organ } & \multicolumn{2}{|c|}{${ }^{111}$ In-DTPA-Tyr ${ }^{3}$-octreotate } & \multicolumn{2}{|c|}{ Cy5-111 In-DTPA-Tyr ${ }^{3}$-octreotate } \\
\hline & Mean \pm SD (\%ID/g) & TTR & Mean \pm SD (\%ID/g) & TTR \\
\hline Blood & $0.02 \pm 0.03$ & 313.84 & $0.07 \pm 0.03$ & 93.72 \\
\hline Spleen & $0.11 \pm 0.03$ & 46.6 & $0.56 \pm 0.04$ & 12.36 \\
\hline Pancreas & $0.29 \pm 0.07$ & 17.99 & $1.90 \pm 0.85$ & 3.65 \\
\hline Liver & $0.12 \pm 0.03$ & 41.97 & $0.51 \pm 0.04$ & 13.57 \\
\hline Kidney & $14.15 \pm 2.79$ & 0.37 & $52.21 \pm 7.85$ & 0.13 \\
\hline Adrenals & $0.40 \pm 0.30$ & 12.99 & $0.62 \pm 0.27$ & 11.1 \\
\hline Duodenum & $0.13 \pm 0.05$ & 38.95 & $0.49 \pm 0.19$ & 14.06 \\
\hline Colon & $0.38 \pm 0.10$ & 13.51 & $1.30 \pm 0.07$ & 5.32 \\
\hline Caecum & $0.17 \pm 0.04$ & 31.28 & $0.43 \pm 0.04$ & 16.28 \\
\hline Stomach & $0.32 \pm 0.06$ & 16.12 & $1.30 \pm 0.31$ & 5.32 \\
\hline Muscle & $0.03 \pm 0.02$ & 168.68 & $0.11 \pm 0.10$ & 61.87 \\
\hline Tumor & $5.16 \pm 1.27$ & 1 & $6.93 \pm 2.08$ & 1 \\
\hline
\end{tabular}

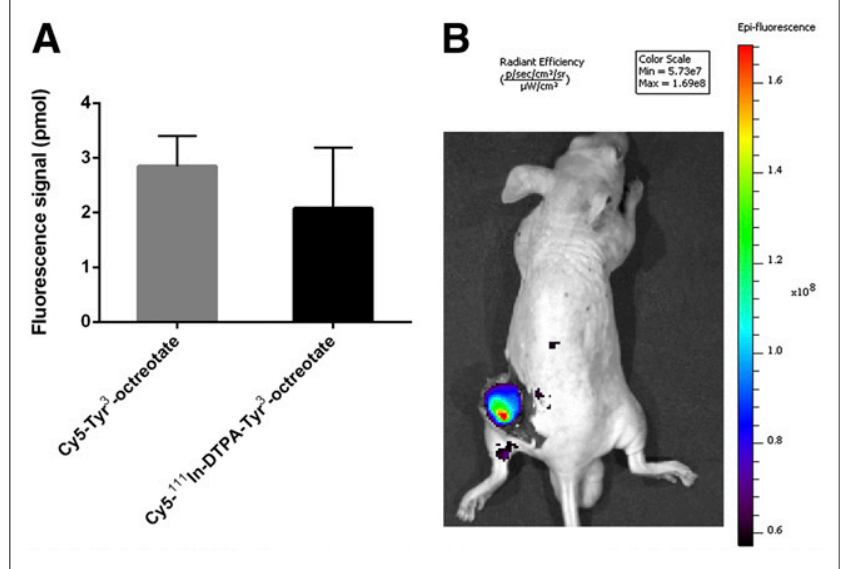

FIGURE 5. (A) FMT-based quantification of Cy5-111 In-DTPA-Tyr ${ }^{3}-$ octreotate and of Cy5-Tyr ${ }^{3}$-octreotate accumulation in tumor at $24 \mathrm{~h}$ after injection. (B) Cy5-Tyr ${ }^{3}$-octreotate fluorescence imaging via IVIS at $24 \mathrm{~h}$ after injection, after removal of skin from tumor area. Max $=$ maximum; $\operatorname{Min}=$ minimum.
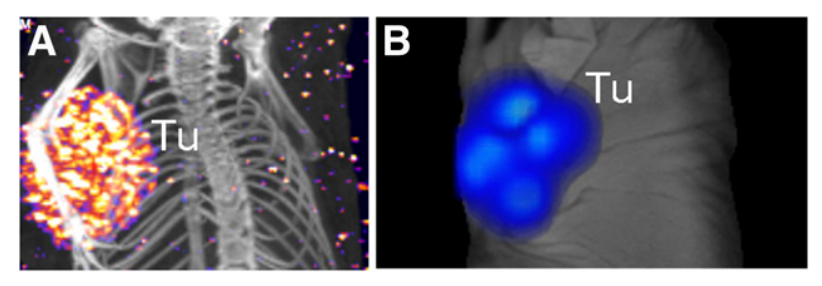

FIGURE 6. Cy5-111In-DTPA-Tyr ${ }^{3}$-octreotate in H69 tumor-bearing mouse $24 \mathrm{~h}$ after injection: SPECT/CT image (A) and FMT image (B) of tumor $(\mathrm{Tu})$ region.

fluorescence guidance (5). In our investigation, the nuclear-based TTR was always greater than 3 (except for the kidneys) and usually greater than 10 . The low liver retention was particularly fa- vorable for NETs, which are known to preferentially metastasize to the liver. To improve the relatively low ( $<1$ TTR) in the kidneys, thereby making identification of tumor lesions near the kidneys feasible, it might be useful to apply methods that have previously been shown to reduce renal uptake of radiolabeled somatostatin analogs, including a hybrid tracer (33).

\section{CONCLUSION}

We have demonstrated that Cy5-111 In-DTPA-Tyr ${ }^{3}$-octreotate can efficiently target $\mathrm{sst}_{2}$ on tumor tissue in vivo, allowing nuclear and fluorescence detection. Our study provides insight into the influence of the Cy5-DTPA label on receptor affinity as well as pharmacokinetics of octreotate in vivo. These findings suggest that this hybrid tracer could be a lead candidate for translation into clinical studies, to achieve more accurate visualization and detection of NETs during surgery.

\section{DISCLOSURE}

The costs of publication of this article were defrayed in part by the payment of page charges. Therefore, and solely to indicate this fact, this article is hereby marked "advertisement" in accordance with 18 USC section 1734. This work used imaging equipment provided by the Applied Molecular Imaging Erasmus MC facility. The work was partially supported by a Dutch Cancer Society translational research award (grant no. PGF 2009-4344), by an NWO-STW-VIDI grant (no. STW-BGT11272), and by the People Program (Marie Curie Actions) of the European Union's Seventh Framework Programme (FP7/2007-2013) under REA grant agreement no. PITN-GA-2012-317019 'TRACE 'n TREAT'. No other potential conflict of interest relevant to this article was reported.

\section{REFERENCES}

1. Reubi JC, Waser B. Concomitant expression of several peptide receptors in neuroendocrine tumours: molecular basis for in vivo multireceptor tumour targeting. Eur J Nucl Med Mol Imaging. 2003;30:781-793. 
2. Laverman P, Sosabowski JK, Boerman OC, Oyen WJ. Radiolabelled peptides for oncological diagnosis. Eur J Nucl Med Mol Imaging. 2012;39(suppl 1): S78-S92.

3. Bison SM, Konijnenberg MW, Melis M, et al. Peptide receptor radionuclide therapy using radiolabeled somatostatin analogs: focus on future developments. Clin Transl Imaging. 2014;2:55-66.

4. Hussain T, Nguyen QT. Molecular imaging for cancer diagnosis and surgery. $A d v$ Drug Deliv Rev. 2014;66:90-100.

5. Tummers QR, Boonstra MC, Frangioni JV, van de Velde CJ, Vahrmeijer AL, Bonsing BA. Intraoperative near-infrared fluorescence imaging of a paraganglioma using methylene blue: a case report. Int J Surg Case Rep. 2015;6C: 150-153.

6. Banerjee SR, Pomper MG. Clinical applications of Gallium-68. Appl Radiat Isot. 2013;76:2-13.

7. Gulec SA, Baum R. Radio-guided surgery in neuroendocrine tumors. J Surg Oncol. 2007;96:309-315.

8. García-Talavera P, Ruano R, Rioja ME, Cordero JM, Razola P, Vidal-Sicart S. Radioguided surgery in neuroendocrine tumors: a review of the literature. Rev Esp Med Nucl Imagen Mol (English ed.). 2014;33:358-365.

9. Wang S, Yang W, Deng J, Zhang J, Ma F, Wang J. Reduction in the recurrence of meningiomas by combining somatostatin receptor scintigraphy of ${ }^{99 \mathrm{~m}} \mathrm{Tc}-$ HYNIC-octreotide SPECT/CT and radio guidance with a hand-held gammaprobe during surgery. Nucl Med Commun. 2013;34:249-253.

10. Lutje S, Rijpkema M, Helfrich W, Oyen WJ, Boerman OC. Targeted radionuclide and fluorescence dual-modality imaging of cancer: preclinical advances and clinical translation. Mol Imaging Biol. 2014;16:747-755.

11. van Leeuwen FW, Hardwick JC, van Erkel AR. Luminescence-based imaging approaches in the field of Interventional molecular imaging. Radiology. 2015;276: $12-29$.

12. Keereweer S, Van Driel PB, Snoeks TJ, et al. Optical image-guided cancer surgery: challenges and limitations. Clin Cancer Res. 2013;19:3745-3754.

13. Azhdarinia A, Ghosh P, Ghosh S, Wilganowski N, Sevick-Muraca EM. Duallabeling strategies for nuclear and fluorescence molecular imaging: a review and analysis. Mol Imaging Biol. 2012;14:261-276.

14. van Oosterom MN, Kreuger R, Buckle T, et al. U-SPECT-BioFluo: an integrated radionuclide, bioluminescence, and fluorescence imaging platform. EJNMMI Res. 2014;4:0056.

15. Van Den Berg NS, Buckle T, Kleinjan GI, et al. Hybrid tracers for sentinel node biopsy. Q J Nucl Med Mol Imaging. 2014;58:193-206.

16. Kuil J, Velders AH, van Leeuwen FW. Multimodal tumor-targeting peptides functionalized with both a radio- and a fluorescent label. Bioconjug Chem. 2010;21: 1709-1719.

17. Edwards WB, Xu B, Akers W, et al. Agonist-antagonist dilemma in molecular imaging: evaluation of a monomolecular multimodal imaging agent for the somatostatin receptor. Bioconjug Chem. 2008;19:192-200.
18. Bunschoten A, Buckle T, Visser NL, et al. Multimodal interventional molecular imaging of tumor margins and distant metastases by targeting alphavbeta 3 integrin. ChemBioChem. 2012;13:1039-1045.

19. Mujumdar RB, Ernst LA, Mujumdar SR, Lewis CJ, Waggoner AS. Cyanine dye labeling reagents: sulfoindocyanine succinimidyl esters. Bioconjug Chem. 1993;4:105-111.

20. Mier W, Eritja R, Mohammed A, Haberkorn U, Eisenhut M. Preparation and evaluation of tumor-targeting peptide-oligonucleotide conjugates. Bioconjug Chem. 2000;11:855-860.

21. de Blois E, Chan HS, Breeman WA. Iodination and stability of somatostatin analogues: comparison of iodination techniques: a practical overview. Curr Top Med Chem. 2012;12:2668-2676.

22. de Blois E, Chan HS, Konijnenberg M, de Zanger R, Breeman WA. Effectiveness of quenchers to reduce radiolysis of ${ }^{111} \mathrm{In}$ - or ${ }^{177} \mathrm{Lu}$-labelled methionine-containing regulatory peptides: maintaining radiochemical purity as measured by HPLC. Curr Top Med Chem. 2012;12:2677-2685.

23. Kuil J, Buckle T, Yuan H, et al. Synthesis and evaluation of a bimodal CXCR4 antagonistic peptide. Bioconjug Chem. 2011;22:859-864.

24. Melis M, Bijster M, de Visser M, et al. Dose-response effect of Gelofusine on renal uptake and retention of radiolabelled octreotate in rats with CA20948 tumours. Eur J Nucl Med Mol Imaging. 2009;36:1968-1976.

25. de Jong M, Breeman WA, Bakker WH, et al. Comparison of ${ }^{111}$ In-labeled somatostatin analogues for tumor scintigraphy and radionuclide therapy. Cancer Res. 1998;58:437-441.

26. Kuil J, Buckle T, Oldenburg J, et al. Hybrid peptide dendrimers for imaging of chemokine receptor 4 (CXCR4) expression. Mol Pharm. 2011;8:2444-2453.

27. Achilefu S, Jimenez HN, Dorshow RB, et al. Synthesis, in vitro receptor binding, and in vivo evaluation of fluorescein and carbocyanine peptide-based optical contrast agents. J Med Chem. 2002;45:2003-2015.

28. Melis M, Krenning EP, Bernard BF, Barone R, Visser TJ, de Jong M. Localisation and mechanism of renal retention of radiolabelled somatostatin analogues. Eur J Nucl Med Mol Imaging. 2005;32:1136-1143.

29. Bunschoten A, Buckle T, Kuil J, et al. Targeted non-covalent self-assembled nanoparticles based on human serum albumin. Biomaterials. 2012;33:867-875.

30. Nock BA, Maina T, Krenning EP, de Jong M. "To serve and protect": enzyme inhibitors as radiopeptide escorts promote tumor targeting. J Nucl Med. 2014;55: 121-127.

31. Krenning EP, Bakker WH, Kooij PP, et al. Somatostatin receptor scintigraphy with indium-111-DTPA-D-Phe-1-octreotide in man: metabolism, dosimetry and comparison with iodine-123-Tyr-3-octreotide. J Nucl Med. 1992;33:652-658.

32. Vaidyanathan G, Affleck DJ, Zhao XG, Keir ST, Zalutsky MR. [Lu]-DOTA-Tyroctreotate: a potential targeted radiotherapeutic for the treatment of medulloblastoma. Curr Radiopharm. 2010;3:29-36.

33. Rolleman EJ, Valkema R, de Jong M, Kooij PP, Krenning EP. Safe and effective inhibition of renal uptake of radiolabelled octreotide by a combination of lysine and arginine. Eur J Nucl Med Mol Imaging. 2003;30:9-15. 